\section{Childhood cancer: trends in incidence and survival}

Because childhood cancers are relatively rare, analysis of trends in incidence depends on largescale, long-term disease registries. Drawing on data collected over 30 years, Steliarova-Foucher and colleagues have recently reported on geographical and temporal patterns of cancer incidence in children and adolescents in Europe. The study shows that the overall incidence of cancer is increasing, and highlights disparities between developed and developing countries in the availability of specialized medical care.

The study included high-quality data on 113,000 tumors in children and 18,243 tumors in adolescents, from 63 population-based cancer registries in 19 European countries. In children under 15 years of age, the overall incidence of cancer rose by $1.0 \%$ per year between 1970-2001, reaching an agestandardized incidence rate of 140 per million in the 1990s (with leukemias, tumors of the central nervous system, and lymphomas being the most frequent). In adolescents, a $1.5 \%$ yearly increase in cancer incidence was recorded-with carcinomas, lymphomas and germ-cell tumors showing the sharpest rise-and the age-standardized incidence rate rose to 157 per million in the same period. Analysis of cancer cases by geographical region showed that overall 5-year survival was higher in the west $(75 \%)$ than in the east (64\%), probably reflecting differences in referral practice and access to costly treatment. Across Europe as a whole, however, survival rates had improved significantly since the 1970 s.

The authors suggest that these findings could be used to evaluate changes in policy and clinical care, provided that the existing database is maintained.

Original article Steliarova-Foucher E et al. (2004) Geographical patterns and time trends of cancer incidence and survival among children and adolescents in Europe since the 1970s (the ACCIS project): an epidemiological study. Lancet 364: 2097-2105

\section{Is MRI a useful adjunct to mammography?}

Magnetic resonance imaging (MRI) of the breast allows detection of lesions not seen by mammography, but the specificity of the technique is in doubt. A recent study has confirmed that MRI, although highly sensitive, does not obviate the need for breast biopsies in patients with suspicious lesions.

Bluemke and colleagues from the International Breast Magnetic Resonance Consortium compared MRI results and subsequent biopsy findings in 821 women with clinically or mammographically suspicious breast lesions to determine whether MRI was a useful adjunct to mammography in these patients. A total of 404 malignant lesions (either ductal carcinoma in situ or invasive carcinoma) were detected histopathologically. Of these, MRI correctly detected cancer in 356 $(88.1 \%)$ cases. Of the 417 patients who did not have cancer, however, MRI results were negative in only 281 cases, yielding a moderate specificity of $67.7 \%$. At $72.4 \%$, the positive predictive value for malignancy for MRI in this study was significantly higher than that for mammography (52.8\%). The negative predictive value for MRI was only $85.4 \%$, however, which is considered to be too low to replace biopsy for suspicious lesions.

The authors add that the performance of MRI appeared to be largely independent of breast density, and so this technique might be useful in patients with dense breast tissue, in whom the sensitivity of mammography tends to be low.

Original article Bluemke DA et al. (2004) Magnetic resonance imaging of the breast prior to biopsy. JAMA 292: 2735-2742

\section{Quantifying risk of recurrence of breast cancer}

A recent paper by Paik and colleagues describes a high-throughput, multigene assay designed to predict recurrence in tamoxifentreated patients with node-negative breast cancer. This might be useful in the development of individualized treatment strategies.

Using data from previous studies, the authors identified a panel of 16 cancer-related genes that showed different levels of expression in patients who did or did not experience recurrence following tamoxifen treatment for breast cancer. They then performed a quantitative RT-PCR assay to measure the levels of 